\title{
Medical Students' Perceptions and Patient Safety Education in China Using the Medical Student Safety Attitudes and Professionalism Survey: Safety attitudes and behavioural intentions
}

He Liu

Harbin Medical University

Dandan Yu

Harbin Medical University

Siqi Zhao

Hebei Medical University

Shufan Sun

institute of psychiatric prevention

mingli jiao

Harbin Medical University

juanjuan Shao

Hebei Medical University

Dan Wang

Hebei Medical University

Min Zhang

Hebei Medical University

yuming Wu ( $\sim$ hebmuwuphy@sina.com)

\section{Research article}

Keywords: Patient safety, medical student, medical education, clinical teaching, organizational safety culture, organizational atmosphere, error reporting, patient safety behaviour, patient safety course

Posted Date: July 23rd, 2019

DOI: https://doi.org/10.21203/rs.2.11835/v1

License: (a) This work is licensed under a Creative Commons Attribution 4.0 International License. Read Full License 


\section{Abstract}

Background Ensuring patient safety is a top priority among health administrators and in the medical communities of all WHO member countries. This study aimed to assess fourth-year medical students' perceptions and experiences with cultural factors during clinical rotation to identify the medical school education needed to promote patient safety. Methods A cross-sectional study was conducted on 615 fourth-year medical students at three medical universities in Heilongjiang Province, China, in 2016 using the Medical Student Safety Attitudes and Professionalism Survey (MSSAPS) questionnaire. Chi-squared for contingency tests compared the percentages of agreement to items across schools and regarding overall patient safety, patient safety behavioural intentions and five cultural dimensions. Spearman correlations assessed statistical associations. Results The five cultural dimensions positively and significantly related to perceptions of overall patient safety and patient safety behavioural intentions. Perceptions of patient safety were positive across the three medical schools. Agreement was almost universal with the following statement: 'We followed standard operating procedures, guidelines, and protocols for the floor' (99.5\%), although $42.8 \%$ agreed that 'A member of my team was rude and disrespectful to a patient or family member'. The respondents' preferences for course content delivery were academic lecture, teaching in clinical rounds and case studies. The preferred course content was 'ways to handle clinical risks', 'the causes of medical errors and the principles of risk prevention' and 'strengthening infection controls and reducing nosocomial infections. Conclusion Fourth-year students seemed willing to learn about this important issue. Policymakers should increase their focus on educational needs based on students' clinical experiences and their emphasis on organizational problems related to error reporting to establish and implement appropriate patient safety education.

\section{Background}

Quality of care and patient safety are the core content and eternal theme of medical services [1]. However, because diseases continue to evolve and change, medical service provision includes risk, continuous learning and a continuous need for improvement. Thus, although lives are being saved and health is being maintained, services include risks to patients' safety.

Patient safety is a global concern, and improving the quality of care and ensuring patient safety has become a focus of the World Health Organization (WHO), its member states, and the global medical community. Specifically, patient safety means that a patient is not harmed during diagnosis and treatment. A 2007 (updated 2008) WHO publication, Ten Facts on Patient Safety, pointed out that patient safety is one of the most common reasons for medical disputes[2]. Statistics of the Chinese Hospital Association indicate that about $66.8 \%$ of patients reported that their safety was not fully protected during medical procedures and that they distrusted them.

Medical educators have asserted that patient safety education is an important part of medical education in the twenty-first century [3]. Patient safety education should start during medical school and all health science colleges and universities should provide relevant courses because medical students represent the 
future of medical care. Establishing an awareness of patient safety issues and cultivating risk prevention and control are vital to constructing a foundation for the future of doctor-patient relationships.

Research on patient safety education in China has lagged behind other countries. The United States (US), Britain, Australia and other countries began to focus on patient safety in about 2000 . For example, The National Institutes of Health (in the US) published Health Professions Education: A Bridge to Quality in 2003 , which suggests six types of core capacities to foster during education with which health practitioners can improve the quality of healthcare services [2]. The British Medical Association takes the position that patient safety education should focus on current pharmaceutical practices in Britain, mechanisms that respond to medical errors and the principles of risk prevention and the ability to provide safe medical services [4]. In Australia, the National Patient Safety Education Framework was published in 2005, and, in 2006, University of Sydney was Australia's first institution to bring patient safety education into its undergraduate healthcare education [5]. In 2008, the Canadian Patient Safety Institute established a multidisciplinary patient safety education framework advocated by medical colleges and institutions as the foundation of developing and continuing courses on patient safety. In 2004, the Japanese government requested medical schools to include education for patients in their undergraduate healthcare courses, and, recently, South Korea and Netherlands have opened their courses to patients. However, there is insufficient emphasis on patient safety in medical education [6].

In October 2004, WHO launched the World Alliance for Patient Safety, followed by a program on patient safety education for medical undergraduates in 2008 [7]. The next year, WHO established a core team of healthcare professionals in Australia and UK, Nie et al. (2011) advocated integrating patient safety into coursework, pre-service patient safety training, and lowering risk by teaching students to regard patients as healthcare partners to reduce friction in the doctor-patient relationship and achieve conceptual consistency. In 2011, WHO implemented an experimental project based on Nie et al. (2011) in 11 medical colleges and universities around the world. Developing countries were prioritized because of their relatively high rate of medical negligence and incidence of accidents.

Places with relatively low medical standards and high medical risks, which is the case in China, are only now starting to attend to the issues [8]. China must quickly implement patient safety education because its patient safety problems are serious. In 2006 through 2007, a WHO/China joint project, entitled Strengthen Patient Safety Management and Education, was conducted to lay the foundation for disseminating patient safety strategies throughout China. Dr Margaret Chan, WHO Director-General from 2006 through 2017, highlighted patient safety as a key to progress [9], which led to the publication of a Chinese version of the WHO Patient Safety Curriculum Guide in July 2012 in support of a Ministry of Health (National Health and Family Planning Commission of the People's Republic of China) resolution.

Only a few studies on patient safety education in China were published until about 2010, which was the pivotal year for such research. In August of 2014, the Chinese Hospital Association published Patient Safety Goals (2014-2015), which reflected in its target clauses and sub-clauses the standards and content of WHO's Patient Safety Actions, the International Patient Safety Standards of the Joint 
Commission International ( $\mathrm{JCl}$ ) Accreditation Standards for Hospitals, Hospital Management Evaluation Guidelines of National Health and Family Planning Commission of the People's Republic of China, Annual Goals on Patient Safety of Taiwan Joint Commission on Hospital Accreditation, and the status of patient safety in China [10]. However, because interest is still new, patient safety education in China lacks research quality and depth, it lacks curricula and training methods and it has been a challenging task to reform medical education to integrate courses on patient safety. This study argues on behalf of patient safety education and the relevant research and activities to support it. The first important information to obtain is the extent of medical students' knowledge of patient safety. Data also are needed regarding students' perceptions of patient safety. In addition, students' explicit educational needs should be assessed and prioritized in a systematic patient safety course of study. Understanding these components is the first step towards providing patient safety education.

In the US, Britain, Australia, Canada and other countries, courses on patient safety emphasize a combination of theory and practice, and they often rely on the practical component as the crucial part of patient safety education [11]. Previous studies have found that the junior (third) year is the best time to teach patient safety. This study employed a sample of senior (fourth-year) students because medical students at this stage have entered clinical practice. The clinical practice stage is a key time for medical students to gain practical skills because they are beginning to shift roles from being a student to being a doctor.

Medical students face a variety of patient safety problems in clinical environments, such as safety competence, safety factors (practical and environmental), risk assessments and prevention, risk situation awareness, error characteristics, reporting adverse events, communications, handling and analysing errors and so on. Educators are increasingly aware that students are influenced by unspoken and hidden cultural factors they experience during clinical rotation as well as what they learn in the classroom. Thus, this study investigated fourth-year students' perceptions of overall patient safety, their patient safety behavioural intentions and five cultural factors. The study's goal was to explore factors considered important to changing students' perspectives on patient safety, addressing students' educational needs, and it aimed to combine China's patient safety education's theoretical research with its practical application.

\section{Methods}

\section{Sample}

A cross-sectional survey was conducted at three purposively selected medical universities (Qiqihar Medical University (QMU), Mudanjiang Medical University (MMU), and University of Jiamusi Medical College (JMU)) in Heilongjiang Province. None of the respondents had previous formal training on patient safety, which enabled the baseline assessment of a safety culture in the sample [12]. Permissions were obtained from the managers of the student offices. Nine hundred questionnaires were distributed to 
fourth-year students, and 615 valid questionnaires were returned for a response rate of $80.6 \%$ (725/900)and total valid rate of $84.8 \%(615 / 725)$.

\section{Questionnaire data}

MSSAPS was used to measure medical students' perceptions of their clinical rotation experiences with respect to safety, teamwork, error disclosure, professionalism, overall patient safety and to assess students' intended safety-related behaviours. First, we obtained permission from Liao to use their questionnaire; then, we translated the questionnaire into Mandarin Chinese and asked 10 experts to revise the questions about its suitability for use in the Chinese culture and the appropriateness of the translation. Two-week test-retest reliability was calculated (0.85) with a group of 40 medical students in three schools. The questionnaire was then back translated into English in order to verify the accuracy of the Mandarin Chinese version.

The questionnaire contained 33 items on six topics as follows:

- Safety culture (items $1-8$ )

- Teamwork culture (items 9-14)

- Error disclosure culture (items 15-18)

- Professionalism culture (items 19-25)

- Expressing professional concerns culture (items 26-28)

- Patient safety behaviour intentions (items 29-33)

We also asked the students the item of 'What overall grade would you give all of your rotations on patient safety?', the item of the overall perceptions of patient safety was measured on a five-point scale (ranging from $A=$ excellent to $E=$ failing) in response.

And the part of the 'Content and Teaching of Patient Safety Education', which contain the items of: The degree of understanding of patient safety; When should patient safety courses be offered at the undergraduate level? What type of patient safety courses should be taught? How many class hours should be taught? What teaching methods should be used take? And what should be included in patient safety courses?

\section{Methods of data analysis}

The responses to the 33 items were on a five-point Likert-type scale where 1 = strongly disagree through 5 = strongly agree. The responses were merged into two groups: responses 1 and $2=$ disagree and responses 4 and $5=$ agree, and the variables distributions were computed [12]. Chi-squared for contingency tests compared schools with respect to agree versus disagree with the 33 items and the cutoff level of statistical significance was 0.05 . Cases with missing date were omitted from the analyses.

\section{Research ethics}


The study design was reviewed and approved by the Research Ethics Committee of Harbin Medical University. Approvals were obtained from QMU, MMU and JMU before the questionnaire was administered. All of the respondents voluntarily and anonymously participated and provided written informed consent.

\section{Results}

Of the 615 respondents, 219 (36\%) were attending QMU, 126 (20\%) were at MMU, and 270 (44\%) were at JMU. About $36 \%(220)$ was male and about $64 \%(395)$ was female. The majority $(69 \%, 424)$ reported that they hoped to further study medicine in China after graduation, and about $88 \%$ preferred to engage in clinical work in the future.

\section{(Insert Table 1 here)}

This study examined variation in five cultural dimensions: safety, teamwork, error disclosure, experiences with professionalism and comfort expressing professional concern as well as patient safety behavioural intentions. Table 2 shows the distributions of responses to the items comprising those constructs. Regarding safety, all except three students agreed that they followed standard operating procedures, guidelines and protocols for the floor. However, only about one-half (53.5\%) of the respondents agreed that 'I was encouraged by colleagues to report any patient safety concerns I may have had', and about three-quarters $(74.4 \%)$ of the sample agreed that 'I knew the proper channels to direct questions regarding patient safety'. Regarding teamwork culture, collaboration with team members and collaboration with nurses (and so on) were strong (95.4\% and $89.4 \%$ agreed, respectively). Despite the opinion that collaboration was 'good', only $67.6 \%$ agreed that 'the quality of care received by patients was influenced by teamwork'.

Regarding error disclosure, $70.4 \%$ agreed that errors were disclosed to patients/families and $75.4 \%$ agreed that it was easy to do so, but only $44.2 \%$ agreed that 'I am encouraged by my colleagues to disclose errors to patients/families'. Agreement with the items on experiences with professionalism was about $50 \%$ or lower on all items. The highest agreement in that group was with the statement that 'one of my superiors behaved inappropriately, but I did not report it because I was afraid it would affect my evaluation' (63.3\%). Less than one-half of the respondents agreed with the statements referring to lowquality professionalism, and they were least likely to agree with the statements that patients were discriminated against (43.1\%) or disrespected (42.8\%). Feeling comfortable expressing professional concerns comprised three items. About three-quarters and nine-tenths of the respondents agreed with expressing concerns and feeling comfortable doing so, respectively. There were five key patient safety behavioural intentions, with which the vast majority of respondents agreed $(71.4 \%$ through $90.7 \%)$. 
Chi-squared for contingency tests revealed statistically significant differences on three of the cultural dimensions (teamwork, error disclosure, and experiences with professionalism) and on patient safety behavioural intentions by school (Table 3 ). JMU respondents were most likely to agree with the teamwork and error disclosure items and they were most likely to agree with the patient safety behavioural intentions' items, whereas they were the least likely to agree with the items comprising experiences with professionalism.

\section{(Insert Table 3 here)}

Correlations were computed among the five cultural dimensions, patient safety behavioural intentions and overall patient safety grade as observed during clinical rotations. All of the cultural dimensions significantly correlated with overall patient safety grade (Table 4 ). The strongest correlation was with the safety construct $(r=.45, p<.05)$ and the weakest was with experiences with professionalism $(r=.13, p<$ .05). The five cultural dimensions also significantly correlated with patient safety behavioural intentions; the strongest relationship was with comfort expressing professional concerns $(r=.53, p<.05)$ and the weakest relationship was with safety $(r=.16, p<.05)$.

\section{(Insert Table 4 here)}

Table 5 implies that about $44.1 \%$ of the sample reported knowing something about patient safety. They were most likely to consider it appropriate to teach patient safety at the intern stage (37.1\%). More than one-half $(55.3 \%)$ reported that patient safety should be a required course as opposed to less demanding alternatives and covered in 20 hours of class time (38.4\%).

\section{(Insert Table 5 here)}

\section{Discussion}


Medical students are future doctors. Studies show that learning about patient safety during their training is more effective than learning on the job [13-14]. Medical students' experiences during their clinical rotations are important influences on their attitudes towards patient safety and their future patient safety behaviours. This study found that medical students' clinical experiences related to their patient safety grade and patient safety behavioural intentions. The results highlight medical students' perceptions of patient safety as an important part of clinical practice. Compared to Lee [15], the three highest scores were for 'We followed standard operating procedures, guidelines, and protocols for the OR'(89.2), 'The quality of care received by patients was impacted by teamwork'(78.4), and 'We followed standard operating procedures, guidelines, and protocols for the floor (e.g. checklists to prev' (73.7). 'Medical error disclosure to patients and families was an important component of patient safety' (73.7), and "I was encouraged by my colleagues to disclose errors to patients/families'(10.3), 'The culture during my rotations made it easy to disclose medical errors'(13.4), 'I received education or training on how to disclose medical errors to patients'(18.0) received the lowest overall scores in that previous study [15]. The current study found that 'We followed standard operating procedures, guidelines, and protocols for the floor (e.g., checklists to prevent central blood stream infections, hand washing)', 'I had good collaboration with team members (students, residents, attending, nurses, and other caregivers)' and 'I observed excellent patient safety practices' were almost universally supported (99.5\%, 95.4\% and 93.7\%, respectively). The statements 'I felt that a patient was discriminated against by a member of my team on the basis of gender, race, sexual orientation, or religion' (43.1\%) and 'A member of my team was rude and disrespectful to a patient or family member' (42.8\%) were strong barriers to positive student patient safety perceptions.

The results found that 'If the superior doctor has misconduct, I will not report it', which was similar to HooYeon Lee, which found that $41.2 \%$ of the sample intended to disclose medical errors committed by team members. The current study's finding might relate to Chinese students' flight from responsibility and pressure caused by unhealthy organizational cultures. Students in the medical school culture struggle to manage and protect themselves in the power structure, and, when mistakes or precursors of mistakes happen, they fear that reporting them might bring poor evaluations down upon them that might negatively influence their futures. Many students do not want to admit that they made a mistake or have a weakness, and they might hesitate to report concerns to avoid problems for their teams. Therefore, we should pay attention to the unwillingness of Chinese students to speak up and increase our vigilance to prevent this fear of exposing defects.

This study revealed that some respondents reported that team members were rude, disrespectful or discriminatory. Sometimes, they inadequately answered patients' questions or ignored them. This is evidence of poor professionalism and professional ethics in healthcare, which previous studies have related to patient safety [16-18]. A culture of professionalism might mitigate errors and procedural violations that primarily arise from cognitive lapses, such as forgetfulness, inattention, low motivation, carelessness or negligence [19-20]. Aside from low levels of personal professionalism, the main reasons for loss of healthcare providers' sense of responsibility might be work intensity or pressure [21-22]. High work intensity and pressure bring to work against the psychological, work numbness, job burnout, 
impatience, gradually evolved into do not care about the questions raised by patients because these questions seem to be less valuable when medical workers are busy to deal with works [23]. Hospital administrators should strive to understand decreasing professionalism and propose appropriate measures for improvement.

Based on the variation among the three schools, QMU should focus on improving its teamwork culture, and MMU should target its safety culture, error disclosure culture and comfort expressing professional concerns. Of the schools, JMU's respondents were most likely to agree with the items, except those about experiences with professionalism, for which they had the lowest agreement. All three schools should aim to improve their curricula in light of their unique weaknesses and continue to improve the cultural factors.

Safety culture was the strongest predictor of the overall patient safety grade, which is consistent with the results of Korean [15] and American [11] studies. A safety culture should be developed to improve the overall extent of patient safety. For example, the extent to which healthcare professionals are comfortable expressing patient safety concerns might influence whether they participate in quality improvement initiatives, report problems, or encourage team members to remember and act on patient safety. We suspect that being comfortable is influenced by the organizational atmosphere because this study's results found that, despite the culture during respondents' rotations made it easy to disclose medical errors, $44.2 \%$ agreed that disclose errors to patients/families will not be too much encouraged by their colleagues. Influenced by the organizational atmosphere, even if one of their superiors behaved inappropriately, they did not report it.

Patient safety education outside China has entered a state-led popularization stage. Classroom teaching is the traditional way of imparting knowledge. Network teaching uses modern tools for distance learning and academic exchange. Simulation is a popular and successful teaching method. Its greatest advantage regarding patient safety is in its ability to transform knowledge and skills into clinical practice. In China, teaching occurs in the classroom and academic lectures. This study's three most popular options for learning patient safety were academic lectures, teaching in clinical rounds, and case studies, and the three most popular topics were 'ways to handle clinical risks', 'the causes of medical errors and the principles of risk prevention', and 'strengthening infection controls and reducing nosocomial infections'.

Because of time and resource limitations, only three institutions participated in this study, which limits its generalizability to medical students in Heilongjiang Province. Additionally, the data were retrospective and, therefore, the results might suffer from the effects of recall bias. However, this study investigated the needs of medical students regarding the structure and content of patient safety courses, which suggests curricular design revisions. Further, few studies have used MSSAPS in China, making this study a valuable foundation for future research.

This study suggests that medical students need targeted courses on patient safety because they consider patient safety an important part of quality of care [11]. These courses would be most effective when students' needs are merged with their preferred course arrangements, although few studies have 
investigated these preferences. This study found that students wanted patient safety education during the intern stage, which might reflect an interest in application during clinical practice.

\section{Conclusions}

Understanding patient safety is vital to good medical practice. This study builds on previous research on Chinese medical students' experiences with patient safety. The results suggest that understanding students' perceptions of patient safety might appropriately guide medical education. The data revealed unprofessional behaviour in clinical practice, and medical professionals should increase their emphasis on organizational problems related to error reporting. Improving patient safety education should be tailored to medical schools' unique strengths and weaknesses, and they should particularly focus on fourth-year medical students during internship.

\section{Declarations}

- Ethics approval and consent to participate

The Institutional Review Board of Harbin Medical University Ethical granted approval before data collection commenced (project identification code: HMUIRB20170016).

- Consent for publication

Not applicable

- Availability of data and material

Data are available from the corresponding author upon request.

- Competing interests

The authors declare that they have no competing interests.

- Authors' contributions

Authors HL, DDY and SQZ equally contributed to this study, all were involved in drafting manuscript; MLJ and YMW designed the study; MZ, JJS and SQZ collected the data; DW and DDY analysed the data. All authors read and approved the final manuscript."

- Acknowledgements

The authors would like to thank all participants in this study. We also thank MLJ and YMW for their guidance on the writing of the manuscript.

\section{References}


1. Kirch D G, Boysen P G. Changing the culture in medical education to teach patient safety[J]. Health Affairs, 2010, 29(9):1600.

2. Aboumatar HJ, Thompson D, Wu A, et al. Development and evaluation of a 3-day patient safety curriculum to advance knowledge, self-efficacy and system thinking among medical students[J]. BMJ Qual Saf, 2012, 21(5): 416-422.

3. Myung SJ, Shin JS, Kim JH, et al. The patient safety curriculum for undergraduate medical students as a first step toward improving patient safety[J]. J Surg Educ, 2012, 69(5): 659-664.

4. Halbach JL, Sullivan LL. Teaching medical students about medical errors and patient safety: evaluation of a required curriculum[J].Acad Med, 2005, 80(6): 600-606.

5. Walton MM, Shaw T, Barnet S, et al. Developing a National patient safety education framework for Australia[J]. Qual Saf Health Care,2006, 15(6): 437-442.

6. Chuang A W, Nuthalapaty F S, Casey P M, et al. To the point: reviews in medical education-taking control of the hidden curriculum[J]. American Journal of Obstetrics \& Gynecology, 2010, 203(4):316.e1.

7. Walton $\mathrm{M}$, Woodward $\mathrm{H}$, Van Staalduinen $\mathrm{S}$, et al. The WHO patient safety curriculum guide for medical schools[J]. Qual Saf Health Care, 2010, 19(6): 542-546.

8. Mingming Zhang,Yurong Duan,Jing Li, et al. Patient safety education:One of the important topics for $21^{\text {st }}$ medical education[J].Chin J Evid-based Med.2010.10(6):637-639.

9. Carruthers S, Lawton R, Sandars J, et al. Attitudes to patient safety amongst medical students and tutors: Developing a reliable and valid measure.[J]. Medical Teacher, 2009, 31(8):370-6.

10. Na Yuan, Chune Liu, Lei Yu. Study Process of Patients Involved in Treatment Decision囚Making State Quo and Influencing Factors [J]. Medicine and Society, 2017, 30(3):58-61.

11. Liao J M, Etchegaray J M, Williams S T, et al. Assessing medical students' perceptions of patient safety: the medical student safety attitudes and professionalism survey[J]. Academic Medicine Journal of the Association of American Medical Colleges, 2014, 89(2):343.

12. Leung G K, Ang S B, Lau T C, et al. Patient safety culture among medical students in Singapore and Hong Kong[J]. Singapore Medical Journal, 2013, 54(9):501-505.

13. Cresswell K, Howe A, Steven A, Smith P, Ashcroft D, Fairhurst K, Bradley F,Magnusson C, McArthur M, Pearson $\mathrm{P}$, et al. Patient safety in healthcare preregistration educational curricula: multiple case study-based investigations of eight medicine, nursing, pharmacy and physiotherapy university courses. BMJ Quality \& Safety. 2013;22(10):843-54.

14. Nie Y, Li L, Duan Y, et al. Patient safety education for undergraduate medical students: a systematic review[J]. Bmc Medical Education, 2011, 11(1):33.

15. Hoo-Yeon Lee, Myung-II Hahm, Sang Gyu Lee.Undergraduate medical students' perceptions and intentions regarding patient safety during clinical clerkship[J].BMC Medical Education (2018) 18:66.

16. Whittemore A D. The impact of professionalism on safe surgical care[J]. Journal of vascular surgery, 2007, 45(2): 415-419. 
17. Levinson W, Yeung J, Ginsburg S. Disclosure of Medical Error.[J]. Jama, 2016, 316(7):764-765.

18. Lee $\mathrm{S} \mathrm{H}$, Phan P H, Dorman T, et al. Handoffs, safety culture, and practices: evidence from the hospital survey on patient safety culture[J]. BMC Health Services Research, 2016, 16(1):1-8.

19. Reason J. Human error: models and management. BMJ. 2000;320:768-70.

20. Shapiro J, Whittemore A, Tsen L C. Instituting a culture of professionalism: the establishment of a center for professionalism and peer support[J]. Joint Commission Journal on Quality \& Patient Safety, 2014, 40(4):168.

21. Aiken $L \mathrm{H}$, Clarke $S \mathrm{P}$, Sloane $D \mathrm{M}$, et al. Hospital nurse staffing and patient mortality, nurse burnout, and job dissatisfaction.[J]. Jama, 1987, 288(16):1987-1993.

22. Panagioti $M$, Panagopoulou E, Bower $P$, et al. Controlled Interventions to Reduce Burnout in Physicians: A Systematic Review and Meta-analysis[J]. Jama Intern Med, 2017, 177(2):195.

23. Dyrbye L N, Shanafelt T D, Sinsky C A, et al. Burnout among health care professionals: A call to explore and address this underrecognized threat to safe, high-quality care[J]. NAM (National Academy of Medicine) Perspective, 2017.

\section{Tables}

Due to technical limitations, tables are only available as a download in the supplemental files section.

\section{Supplementary Files}

This is a list of supplementary files associated with this preprint. Click to download.

- Table15.pdf 